\title{
A THEORETICAL STUDY ON PARTIALLY AUTOMATED METHOD FOR (PROSTATE) CANCER PINPOINT USING MAGNETIC RESONANCE IMAGING (MRI)
}

\author{
M.Venkatesh Kumar ${ }^{1}$, P.Kalamani ${ }^{2}$, M. ChithambaraThanu ${ }^{3}$ \\ ${ }^{1}$ Sr. Lecturer, ${ }^{2}$ Asst. Prof, ${ }^{3}$ Asst. Prof.,Dept. Of CSE, SSEC, Bangalore, India.
}

\begin{abstract}
A Partially Automated method for (Prostate) Cancer pinpoint using Multi-parametric magnetic resonance imaging has been proposed in this paper, which can be used in guiding surgery. A Random Walker $(R W)$ algorithm has been analyzed with seed initialization to perform (Prostate) cancer pinpoint using Magnetic Resonance Imaging (MRI). Segmentation can be done by using Random Walker $(R W)$ algorithm which has to be considered to be a fastest method. Random Walker (RW) method can be used with multi-parametric magnetic resonance imaging (MRI) and then by using Support Vector Machine (SVM) method, we can determine the seed points in a partially automated manner. By using this method, more weights to the image can be assigned in order to produce improved segmentation process. The proposed method can also give high specificity rate without reducing the sensitivity which is better than earlier methods and fisher sign test can be also used to find the statistical differences.
\end{abstract}

Index terms:Support Vector Machine, Random Walker, Magnetic Prediction, Magnetic Resonance.

\section{INTRODUCTION}

A (Prostate) cancer develops in a gland i.e. male reproductive system. It is one of the typical cancers which spreads in other parts of the human body especially bones and lymph nodes. Pain while urinating, sexual intercourse and erectile dysfunction are some of the symptoms of this cancer. Initial treatment is more important which the prostate specific antigen testing increases cancer detection, but does not decreases mortality [3], [10].
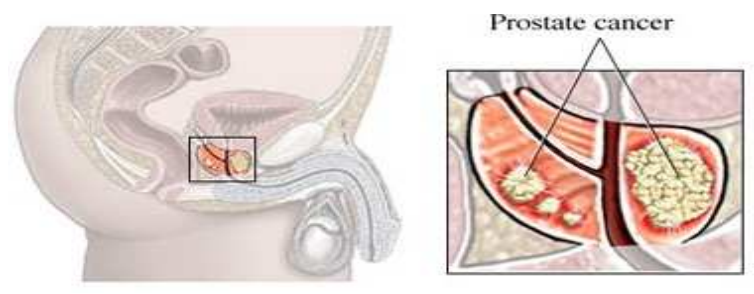

Fig-1: Prostate Cancer in human body

\subsection{Prostate Imaging}

The imaging methods used for (Prostate) cancer detection are Ultrasound (US) and Magnetic Resonance Imaging (MRI). The main drawback of Ultrasound (US) technique is poor tissue resolution, which cannot be used clinically. But compared to Ultrasound (US), MRI (Good Soft tissue Resolution) uses magnetic fields to locate and characterize (prostate) cancer [7], [19]. Multi-parametric prostate Magnetic Resonance Imaging (MRI) consists of 4 types which are follows:

1. Weighed Imaging (WI).

2. Diffusion Weighed Imaging (DWI).
3. Magnetic Resonance Spectroscopic Imaging (MRSI).

4. Dynamic Construct Enhanced Imaging (DCEI).

\section{LITERATURE SURVEY}

\section{Accurate Automatic Analysis of Cardiac Cineimages}

In this automated approach, they are 3 steps for analyzing the thickness and thickening of the images. First step is to segment then inner and outer wall tissue borders by using the geometric deformable model. In Second step, using Laplace equation, point-to-point correspondence between inner and outer borders of the tissue was found. In last step, Gauss Markov Random Field (GGMRF) model is used to reduce the errors. In this approach, the segmentation is based upon the ROC (Receiver Operating Characteristic) and DSC (Dice similarity Coefficients for higher accuracy [12] - [25].

\section{EXISTING METHOD \& DRAWBACKS}

In earlier method, manual and fully (i.e. supervised and unsupervised) segmentation had been performed using multi-parametric MRI. By using semi-supervised cancer pinpoint, labeling of data requires laborious human annotation. According to Tiwari et al., representation of individual data is combined multi-kernel which is followed by semi-supervised dimensionally reduction, incorporated to a high dimensional data in a reduced space. 


\subsection{Drawbacks of Existing system}

In previous method, Weighed and Apparent diffusion coefficient method is used instead of Random Walker (RW) and Support Vector Machine (SVM) method for prostate cancer localization [Artan et al.]. Manual seeds are initialized rather than all the multi-parametric images.

\section{PROPOSED METHOD \& ADVANTAGES}

In the proposed method, Random Walker (RW) technique can be used for image segmentation which is used in multiparametric images. This method combines multiple image segmentation [Wattaya et al.] results in which each can be obtained by using Random Walker (RW) algorithm after varying the number of user assigned seeds. Then it is compared with Locality preserving projections (LPP) method of [Grady et al.] and [Levin et.al] for edge weights by applying linear transformation to RGB color.

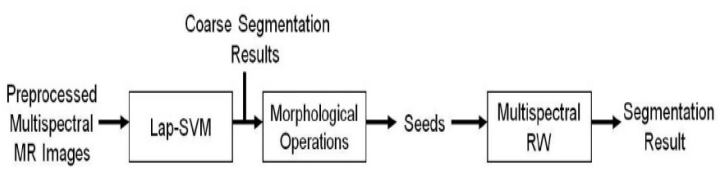

Fig- 2: Segmentation Process

\subsection{Advantages of Proposed Method:}

- To identify (Prostate) cancer pinpoint with multiparametric MRI.

- Automated Seed initialization for RW algorithm.

- Several MR image types are combined optimally and automate the seed generation process for RW algorithm using discriminative learning techniques.

\subsection{Algorithm}

Step1:Laplacian-Support Vector Machine (Lap-SVM) is used to produce a rough estimate on tumor locations for a given a set of multi-parametric MR images.

Step2:Isolated pixels are removed from Lap-SVM (Laplacian-Support Vector Machine) output, and eccentricity values are determined for each of the connected components in the resulting binary image

Step 3: Applying erosion operation on the binary image.

Step4:Assigning a positive seed at the center of the remaining connected component(s) and a negative seed to the location corresponding to the minimum Lap-SVM (Laplacian-Support Vector Machine) value in the unthresholded Lap-SVM (Laplacian-Support Vector Machine) output.

\section{ARCHITECTURE}

In previous work, segmentation method is designed by combining conditional random fields (CRF) with a costsensitive SVM, which allowed incorporating spatial information in the segmentation process. Incorporation of spatial information is achieved through seed points that are selected via SVM (Support Vector Machine) in the proposed method. These seed points allow us to accurately localize desired regions, higher specificity and sensitivity rates compared to (seeded) spatial algorithm in our segmentation scheme.

\section{List of Modules:}
1. Normalization
2. Anisotropic Filtering
3. Multi-parametric $R W$
4. Seed Generation using Laplacian SVM

\subsection{Normalization}

Multi-parametric MRI dataset can be used, which can consist of three different types of MR images. Each multiparametric component represents a particular anatomical and functional response of the prostate gland. Feature vectors used in our segmentation framework are the intensity values of the multi-parametric MR images. The prostate consists of various zones such as CG (Central Gland) and PZ (Peripheral Zone). PZ (Peripheral Zone) region is located at the back of the prostate gland, which is close to the rectum [23-27].

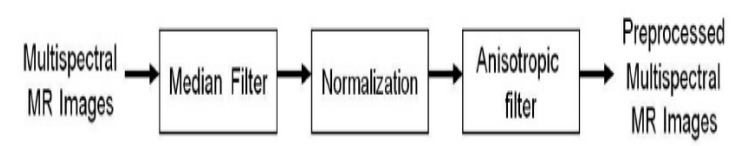

Fig-3: Normalization process

For each of the multi-parametric images, PZ (Peripheral Zone) region intensities were normalized such that intensities in the PZ (Peripheral Zone) region had zero mean and unit standard deviation for all the training and testing subjects for a particular multi-parametric image type. This process brings intensities of different types of MR (Magnetic Resonance) images within the same dynamic range, improving the segmentation methods [5], [10], [11].

\subsection{Anisotropic Filtering}

Magnetic resonance images are typically corrupted by thermal noise due to receiver coils. Therefore, many earlier studies proposed various filtering schemes to remove noise before doing any further processing on them [30]. However, while suppressing the noise, we want to simultaneously preserve the information-bearing structures such as edge boundaries. Anisotropic filtering allows us to smooth PZ (Peripheral Zone) regions of multi-parametric images without blurring the tumor nodule edges. The diffusion equation for an image $u$ is given by,

$$
\frac{\partial u}{\partial t}=\operatorname{div}(\rho(|\nabla u|) \cdot \mid \nabla u)
$$

Equation encourages smoothing within PZ (Peripheral Zone) region while preserving tumor boundaries. This method will not cause interregional blurring as often caused 
by traditional smoothing techniques. Note that anisotropic filtering needs to be applied on the median-filtered and normalized images for a successful application as noted in our earlier study [25-29].

\subsection{Multi-Parametric RW}

RW (Random Walker) algorithm is a seeded segmentation technique that formulates the classical segmentation problem in terms of a discrete combinatorial problem. An edge connecting two vertices is denoted as $\boldsymbol{e i j}$. A weighted graph has a value assigned to each edge called a weight denoted by wij. In the original RW formulation and used the typical Gaussian weighting function given

$$
w_{i j}=\exp \left(-\beta\left(g_{i}-g_{j}\right)^{2}\right)
$$

by,

Where, $g i$ indicates the image intensity at pixel $i$, and the value of the scalar is selected based on experience. Linear weighted combination of features yields a final image that yields improved RW (Random Walker) segmentation for the given set of seeds. Edge weights for the multi-parametric problem $\boldsymbol{w i j}$ can now be written as,

$$
w_{i j}=\exp \left\{-\beta\left(\sum_{n=1}^{N} k_{n}\left(g_{n i}-g_{n j}\right)\right)^{2}\right\} .
$$

\subsection{Seed Generation Using Laplacian SVM}

In this algorithm, the Seeds are manually initialized. Therefore, we have compared various methods, namely, support vector machines (SVM), transductive support vector machines (TSVM) and Laplacian support vector machines (Lap-SVM), to develop a seed selection technique most suited for (prostate) cancer pinpoint. SVM (Support Vector Machine) method is used only for seed generation for the RW algorithm, not the actual segmentation [15]. The geometry of data is modeled as a

graph in which nodes represents the labeled and unlabeled samples connected by weights wij resulting,

$$
\|f\|_{M}^{2}=\frac{1}{(\ell+u)^{2}} \sum_{i, j=1}^{\ell+u} w_{i j}\left(f\left(\mathbf{x}_{i}\right)-f\left(\mathbf{x}_{j}\right)\right)^{2}
$$

\section{CONCLUSIONS}

We presented a framework for automatically localizing prostate cancer with multi-parametric MR images using a new approach of partially supervised segmentation in this paper. Proposed Random Walker [RW] method produce improved segmentation results by assigning more weights to the images. Our study show that significantly improved segmentation results could be obtained with the proposed method compared to earlier developed methods. Fisher's sign test can be also used to show improvements with our method are statistically significant. Multiple initializations for the optimum values increases the computation cost slightly, and decreasing this computation is a subject of our future study.

\section{REFERENCES}

[1] American Cancer Society, Surveillance and Health Policy Research, 2010.

[2] B. Turkbey, P.S. Albert, K. Kurdziel, P. L. Choyke, "Imaging localized prostate cancer: current approaches and new developments," American Journal of Roentgenology, vol. 192, no. 6, pp. 1471-1480, June 2009.

[3] H. Ito, K. Kamoi, K. Yokoyama, K. Yamada, T. Nishimura, "Visualization of prostate cancer using dynamic contrast-enhanced MRI: comparison with transrectal power doppler ultrasound," British Journal of Radiology, vol. 76, no. 909, pp. 617-624, Sep. 2003.

[4] F. A. van Dorsten, M. van der Graaf, M.R. Engelbrecht, G.J. van Leenders, A. Verhofstad, M. Ripkema, J. J. de la Rosette, A. Heerschap, "Combined quantitative enhanced MR imaging and (1)H MR spectroscopic imaging of human prostate cancer," Journal of Magnetic Resonance Imaging, vol. 20, no. 2, pp. 279-287, Aug. 2004.

[5] J. Futterer, S. Heijmink, T. W. Scheenen, J. Veltman, H. J. Huisman, Vos P, C.A. Hulsbergen-Van de Kaa, J. A. Witjes, P. F. Krabbe, A. Heerschap, J. O. Barentsz, "Prostate cancer localization with dynamic contrastenhanced MR imaging and Proton MR spectroscopic imaging," Radiology, vol. 241, no. 2, pp. 449-458, Nov. 2006.

[6] N. Altaf, L. Daniels, P. Morgan, J. Lowe, J. Gladman, S. MacSweeney, A. Moody, and D. Auer, "Cerebral white matter hyperintense lesions areassociated with unstable carotid plaques," Eur. J. Vasc. and Endovasc. Surg., vol. 31, pp. 8-13, 2006.

[7] Z. Lao, D. Shen, A. Jawad, B. Karacali, D. Liu, E. Melhem, R. Bryan, and C. Davatzikos, "Automated segmentation of white matter lesions in 3D brain MRI, using multivariate pattern classification," in Proc. IEEE Int. Symp. Biomed. Imaging (ISBI), 2006, pp. 307-310.

[8] P. Anbeek, K. L. Vincken,M. J. van Osch, R. H. Bisschops, and J. van der Grond, "Automatic segmentation of different-sized white matter lesions by voxel probability estimation," Med. Image Anal., vol. 8, no. 3, pp. 205- 215, 2004.

[9] P. Anbeek, K. Vincken, M. van Osch, R. Bisschops, and J. van der Grond, "Probabilistic segmentation ofwhite matter lesions inMRimaging," NeuroImage, vol. 21, no. 3, pp. 1037-44, 2004.

[10] R. de Boer, F. van der Lijn, H. A. Vrooman,M.W. Vernooij, M. A. Ikram, M. M. Breteler, andW. J. Niessen, "Automatic segmentation of brain tissue andwhite matter lesions in MRI," in Proc. IEEE Int. Symp. Biomed. Imag., 2007, pp. 652-655. 
[11] R. de Boer, H. A. Vrooman, F. van der Lijn,M.W. Vernooij, M. A. Ikram, A. van der Lugt, M. M. Breteler, and W. J. Niessen, "White matter lesion extension to automatic brain tissue segmentation on MRI," Neuroimage,vol. 45, no. 4, pp. 1151-1161, 2009.

[12] R. Khayatia, M. Vafadusta, F. Towhidkhaha, and S.M. Nabavib, "Fully automatic segmentation of multiple sclerosis lesions in brain $\mathrm{mr}$ flair images using adaptivemixturesmethod andMarkov random field model," Comput. Biol. Med., vol. 38, no. 3, pp. 379-390, 2008.

[13] R. Khayatia, M. Vafadusta, F. Towhidkhaha, and S. M. Nabavib, "A novel method for automatic determination of different stages of multiple sclerosis lesions in brain mr flair images," Comput. Med. Imag. Graph., vol. 32,no. 2, pp. 124-133, 2008.

[14] F. Barkhof and R. Smithuis. (2007). "Multiple sclerosis," Radiology Assistant, [Online]. Available: http://www.radiologyassistant.nl/en/4556dea65db62

[15] C. R. Jack, P. C. O’Brien, D. W. Rettman, M. M. Shiung, Y. C. Xu, R. Muthupillai, A. Manduca, R.Avula, andB. J. Erickson, "Flair histogram segmentation for measurement of leukoaraiosis volume," J. Mag. Res. Img., vol. 14, no. 6, pp. 668-676, 2001.

[16] K. V. Leemput, F. Maes, D. Vandermeulen, and P. Suetens, "A unifying framework for partial volume segmentation of brain MR images," IEEE Trans. Med. Imag., vol. 22, no. 1, pp. 105-119, Jan. 2003.

[17] M. A. Gonz'alez Ballester, A. P. Zisserman, and M. Brady, "Estimation of the partial volume effect in MRI," Med. Image Anal., vol. 6, no. 4, pp. 389-405, 2002.

[18] A. Khademi, D. Hosseinzadeh, A. Venetsanopoulos, and A. R. Moody, "Nonparametric statistical tests for exploration of correlation and nonstationarity in images," in Proc. Int. Conf. Digital Signal Process., 2009, pp. 1-6.

[19] A. Khademi, A. Venetsanopoulos, and A. R. Moody, "Automatic contrast enhancement of white matter lesions in FLAIR MRI," in Proc. IEEE Int. Symp. Biomed. Imag., 2009, pp. 1-4.

[20] P. Meer and B. Georgescu, "Edge detection with embedded confidence," IEEE Trans. Pattern Anal. Mach. Intell., vol. 23, no. 12, pp. 1351-1365, Dec. 2001.

[21] A. Khademi, A. Venetsanopoulos, and A. Moody, "Edge-based PVA estimation in FLAIR MRI with WML," in Proc. IEEE Eng. Med. Biol. Soc. Conf., 2010, pp. 61146117.

[22] P. H. Kvam and B. Vidakovic, Nonparametric Statistics with Applications to Science and Engineering. New York: Wiley-Interscience, 2007.

[23] A. Popovic, M. de la Fuente, M. Engelhardt, and K. Radermacher, "Statistical validation metric for accuracy assessment in medical image segmentation," Int. J. Comput. Assist. Radiol. Surg., vol. 2, pp. 169-181, 2007.
[24] C. Lisanti, C. Carlin, K. P. Banks, and D.Wang, "NormalMRI appearance and motion-related phenomena of CSF," Amer. J. Roentgenol., vol. 188, no. 3, pp. 716-725, 2007.

[25] Vijayakumar, B., and Ashish Chaturvedi. "Automatic Brain Tumors Segmentation of MR Images using Fluid Vector Flow and Support Vector Machine." Research Journal of Information Technology 4.

[26] Vijayakumar, B., and Ashish Chaturvedi. "Tumor CutSegmentation and Classification of MR Images using Texture Features and Feed Forward Neural Networks." European Journal of Scientific Research 85.3 (2012): 363372

[27] Vijayakumar, B., and Ashish Chaturvedi, "Idiosyncrasy Dissection and Assorting of Brain MR Images Instigating Kernel Corroborated Support Vector Machine," Archive Des Science [28] Vijayakumar, B., and Ashish Chaturvedi, "Abnormality segmentation and classification of brain MR images using Kernel based Support vector machine," Archive Des Science, Volume 66,Issue 4.

[29] B. Vijayakumar, Ashish Chaturvedi and K. Muthu Kumar, Brain Tumor in Three Dimenesional Magnetic Resonance Images and Concavity Analysis, International Journal of Computer Application, Issue 3, Volume 1 (February 2013),

[30] B. Vijayakumar, Ashish Chaturvedi and K. Muthu Kumar, Effective Classification of Anaplastic Neoplasm in Huddling Stain Image by Fuzzy Clustering Method, International Journal of Scientific Research, Issue 3 volume 2, March-April 2013. 